\title{
Dynamic Control of Interference Effects between Optical Filaments through Programmable Optical Phase Modulation
}

\author{
R. Borrego-Varillas, J. Pérez-Vizcaíno, O. Mendoza-Yero, \\ J. R. Vázquez de Aldana, G. Mínguez-Vega, and J. Lancis, Member, IEEE.
}

1

\begin{abstract}
Light beams shaped by programmable megapixel spatial light modulators (SLMs) are key to broaden the applications of photonics. In this paper, we consider the application of a SLM for the generation of two mutually coherent white-light continuum optical sources by filamentation of infrared femtosecond pulses in bulk. We demonstrate that the inhomogeneity of the input beam and the longitudinal separation of the generated filaments are crucial parameters that break down the mutual coherence across neighboring filaments. We show that local control over the optical phase enables us to gain fine control over filament interference effects.
\end{abstract}

Index Terms - Optical pulse shaping, spatial coherence, supercontinuum generation.

\section{INTRODUCTION}

$\mathrm{S}_{\mathrm{s}}^{\mathrm{u}}$ UPERCONTINUUM (SC) generation refers to an extreme spectral broadening, which is usually accomplished by strong nonlinear propagation of a laser pulse [1]. It has been observed in a variety of media ranging from gases and liquids, to solids [2], including photonic crystal fibers [3]. Thanks to its broadband and ultrashort characteristics, it is a unique light source for practical applications such as femtosecond timeresolved spectroscopy [4], spectral interferometry [5], or its use as seed pulses for optical parametric amplifiers (OPAs) [6]. One mechanism to generate SC with ultra-short pulses is based on filamentation in transparent media [7]. In filamentation, the pulse experiences a complex nonlinear propagation in which self-focusing (optical Kerr effect) and laser-induced ionization dynamically interact to produce self-guiding of the pulses for distances larger than the Rayleigh length. As a result, an extreme spectral broadening (SC) is typically produced. Filamentation in bulk and gases is a widespread technique for ultrashort pulse generation at moderate and high intensities [8].

This research was funded by Generalitat Valenciana through the programme (PROMETEO\ 20121021), Spanish Ministry of Science (FIS2013-40666-P) and University Jaume I through the project P1 1B2013 - 53. The authors are also very grateful to the SCIC of the Universitat Jaume I for the use of the femtosecond laser.

R. Borrego-Varillas, J. Pérez-Vizcaíno, O. Mendoza-Yero, G. MínguezVega, and J. Lancis, are with the Departament de Ciències Experimentals, Universitat Jaume I, 12080 Castelló, Spain (e-mail: rborrego@uji.es).

J. R. Vázquez de Aldana is with the Grupo de Investigación de Microprocesado de Materiales con Láser, Universidad de Salamanca, E-37008, Spain.
Moreover, there are diverse applications for SC sources which strongly depend upon coherence of the SC source, such as optical coherence tomography [9], high precision metrology [10], high-speed telecommunication industry [11], ultrashort pulse generation [12] and accuracy frequency measurement in spectroscopy [13]. Furthermore, the spectral coherence properties of a light pulse have shown to play an important role in the product achieved from some chemical reactions in a pump-dump scenario [14].

The coherence properties of the SC have been the subject of diverse investigations, in both bulk materials as photonic crystal fibers. In physical systems governed by nonlinear dynamics, any tiny variation of the input magnitudes leads to a significant variation at the output. The seminal paper of Dudley [15] set the basis for a proper description of the stochastic nature of the SC generated in photonic crystal and tapered optical fibers. More recently, the detailed coherence properties of SC radiation generated in nonlinear fibers were described using second-order coherence theory. In this way it was shown that SC can be divided into quasi-coherent and quasi-stationary parts and that the relative contributions depend on the dynamics involved in the spectral broadening $[16,17]$.

Concerning SC pulses generated in bulk media, Bellini and Hänsch were the first to demonstrate that two white-light continuum pulses that are independently generated by phaselocked ultrashort pulses are locked in phase [18]. The mutualtemporal coherence of SC generated in glass was experimentally investigated using a diffraction-grating-based interferometer [19] and has been also demonstrated using collinear geometries with time-delayed pump pulses [20]. Watanabe et al. studied the coherence of an array of continuum sources generated by focusing 130 fs pulses in water [21], concluding that they were mutually coherent. Baum et al. demonstrated stable interference between the outputs of two noncollinearly phase-matched optical parametric amplifiers seeded by separate continua beams [22]. Stable interferences between the infrared and visible parts of a continuum spectrum have also been measured [23], evidencing that both of them are locked in phase. The coherence properties of white-light have also been exploited to generate variable linear arrays of phasecoherent SC sources [24] and it has been demonstrated the generation of a regular array of white-light filaments in glass by the use of diffractive microlenses [25]. 
The coherence of two adjacent SC sources is thus an issue of great importance in multi-beam filamentation. In fact, the coherence among filaments influences the ability of them to interact. Various interaction scenarios, such as attraction, repulsion, fusion, spiral motion, amplification, and energy exchange among the interacting filaments were demonstrated depending on the interaction geometry, input power, time delay, relative phase, and beam separation [26]. Additionally, small variations of the degree of coherence may also result in a qualitative change in the emission pattern of the continuum [27]. The tunability in the emission of the white-light continuum allows us to modify the supercontinuum for spectroscopic applications such as remote sensing [28].

Under certain circumstances, the coherence can be measured with a Young's two pinhole interference setup. The intimate relation between the phase correlations of the fields at the two pinholes and the visibility of the interference fringes relies on spatial and temporal overlapping of the beams. This limitation, which often is not explicitly stated, severely restricts the use of Young's interferometer for coherence measurement of multiple SC sources. The reason is the lack of homogeneity of amplified femtosecond beams, which are usually characterized by a poor beam quality factor (higher than 2). This leads to temporal delay between the beams that makes it impossible to observe interference effects at practical geometries.

On the other hand, liquid-crystal displays working as electronically addressed spatial light modulators (SLMs) have been used to generate programmable diffractive lenses. Current megapixel SLMs allow for a fine control over the local phase of an incident beam on real time. This can compensate for potential aberrations and spatial inhomogeneities of the beam. Moreover, the refresh rates of current SLMs allow for real-time control over the focal length and efficiency of the diffractive lenses. In spite of these advantages, the use of SLMs for SC coherence measurements has not been yet reported.

In this paper, we show a way of changing and optimizing, in real time, the visibility of the interference fringes between two SC sources produced by filamentation in bulk (fused silica). For this purpose, two microlenses are implemented onto a SLM, thus allowing for a precise control of the focus position and the energy coupled into each filament. The weak plasma emission generated in the filaments allows us to image them onto a CCD and check with precision their longitudinal positions.

The paper is organized as follows: in section II we use a standard interferometer that allows us to better understand the interference effects between two adjacent SC sources, generated by focusing two infrared beams in a fused silica sample. In this section we also discuss the loss of coherence as the filaments are separated along the longitudinal axis. This is crucial to understand the role that the separation between the sources can cause on fringe visibility when the SLM is employed. In such case, two lenses are implemented on the SLM (section III), but the beam inhomogeneities may lead the two beams to self-focus at slightly different positions inside the sample. In section IV we investigate this problem and propose an approach to account for this effect so that the SLM can be used to control filament interference effects. Finally, the main conclusions are presented in section $\mathrm{V}$.

\section{THEORETICAL AND EXPERIMENTAL BACKGROUND}

In order to fully characterize the properties of the mutual coherence between two adjacent SC sources in a conventional scenario, as a first step, we built a Mach-Zehnder interferometer (Fig. 1). It allowed us some versatility because we can independently vary the geometrical separation and the temporal delay of the two focused pulses. This enables us to investigate the mechanisms behind the loss of coherence under certain circumstances.

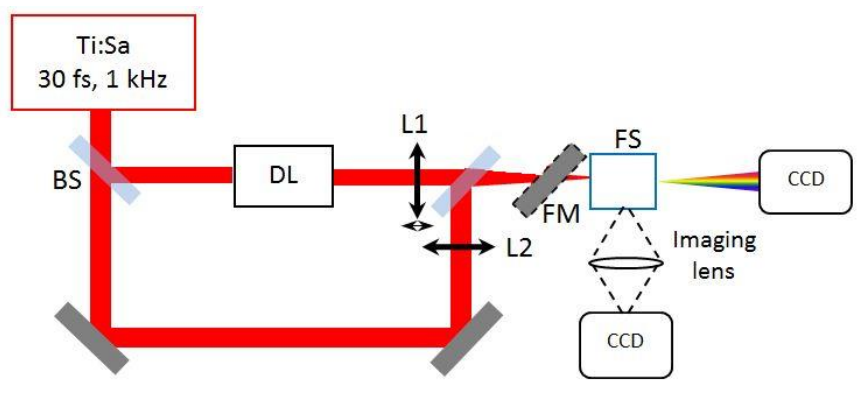

Fig. 1. Mach-Zehnder interferometer (BS: beam splitter; DL: delay line; DP: diffusing plate; FM: flip mirror; FS: fused silica plate; L1 and L2: refractive lenses).

As a light source we used a Ti:Sapphire femtosecond laser that emits linearly-polarized pulses of about $30 \mathrm{fs}$ full-width at half maximum (FWHM) in intensity, centered at $\lambda_{0}=800 \mathrm{~nm}$, with a spectral bandwidth of $50 \mathrm{~nm}$ FWHM and a repetition rate of $1 \mathrm{kHz}$. The energy was controlled by means of a variable attenuator. A $7 \mathrm{~mm}$ iris was placed in front of the interferometer (this will allow us to keep the same numerical aperture that in our second experiment which includes an SLM). The beam was then split in two replicas, each one propagating in the different arms of the interferometer. In order to separate the beams laterally at the output, one of the replicas was aligned at a slightly different height than the other. Two identical refractive lenses $(\mathrm{f}=150 \mathrm{~mm})$ were inserted in both arms respectively: the first lens was fixed, whereas the other was mounted in a translational stage to finely vary the separation between foci. Additionally, one of the arms was equipped with a retroreflector mounted in a translational stage, which allowed us to control the temporal delay between pulses.

The target material for SC generation was a $5-\mathrm{mm}$ thick fused silica sample. The energy was set to $\sim 0.5 \mu \mathrm{J}$ per arm (under our experimental conditions, thresholds for SC generation in this sample were $0.40 \mu \mathrm{J}$ in one arm and $0.38 \mu \mathrm{J}$ in the other). When the geometrical foci for both lenses were situated at the same depth of the sample, we obtained two identical filaments laterally separated by $0.22 \mathrm{~mm}$ (Fig. 2a). Well-contrasted fringes appeared when both pulses were temporally overlapped, i.e. $t=0$ fs. The profile of the corresponding images registered by the CCD is shown as an inset in Fig. 2a (bottom). From the fringes generated by the 
interference of the two SC beams we can measure their visibility [29]

$$
V=\frac{I_{\max }-I_{\min }}{I_{\max }+I_{\min }}
$$

In our case, we observed that for the equal optical paths and zero delay our SC sources had a visibility of about 0.80 . This was a typical value as it is well known from the high coherence of SC beams [18-21].
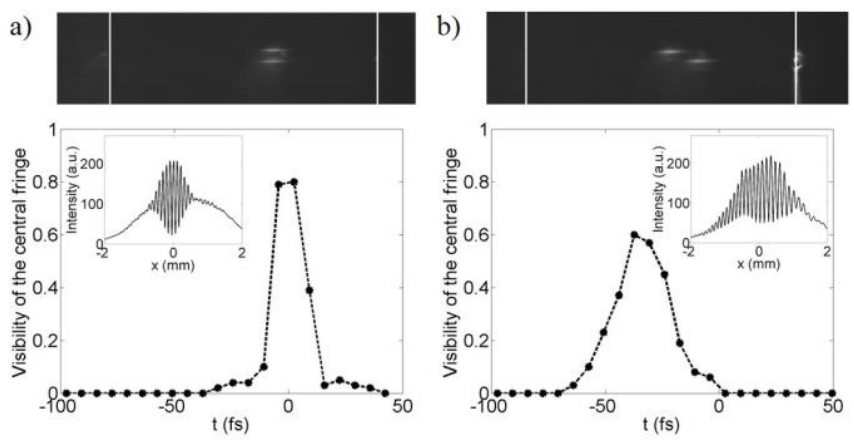

Fig. 2. Panel a) corresponds to the case where one of the both geometrical foci rely on the same plane, whereas in b) one was slightly displaced. Top: images of filaments profiles. Bottom: visibility of the central fringe as a function of the time delay. Insets show the spatial fringes extracted from the interferogram.

The bottom part of Fig. 2a represents the visibility of the central fringe as a function of the delay between pulses, $\mathrm{V}(\tau)$. Since interferences will only develop at those points where pulses temporally overlap, this curve can be understood as a cross-correlation between SC pulses coming from the two adjacent filaments. The width of such curve, $\Delta \tau$, is therefore related to the SC pulse duration, and consequently to the coherence length. Under these experimental conditions, we found $\Delta \tau=17.0$ fs (FWHM).

If the two foci were displaced $0.6 \mathrm{~mm}$ along the propagation axis by moving one of the lenses (Fig. 2b), we observed that no fringes appeared at $t=0$ fs. Instead, the highest contrast was found at $\mathrm{t}=-37 \mathrm{fs}$. This temporal separation of the pulses can be partially attributed to the group velocity mismatch (GVM) between infrared (input pulses) and visible SC pulses, together with the different divergences of the SC and infrared beams. On the other hand, the maximum value of visibility is lower, $\mathrm{V}=0.6$, than that obtained when the foci are in the same plane. We explain this difference on the basis of spectral densities: since filaments develop at different depths within the material, the intensity of the spectral components is not equalized, thus affecting the fringe contrast. The width of the curve visibility vs. delay was also larger than in the previous case, $\Delta \tau=67.0 \mathrm{fs}$. We attribute this behavior to pulse lengthening due to chromatic dispersion: the SC generated in the second filament will suffer material dispersion for $0.6 \mathrm{~mm}$ more than the other one. As a consequence, the pulse is chirped and thus lengthened in time. In summary, we have observed that when the filaments are displaced along the propagation axis, no fringes appeared unless the delay between pulses is adjusted, at the expense of the reduction of the visibility. However, the non-uniform beam profile of amplified femtosecond beams may lead to the generation of two displaced filaments when using the SLM, a drawback to be overcome.

\section{EXPERIMENTAL SETUP}

Once we have studied the SC sources generated with a conventional interferometer, we focus our attention in the main objective of the paper that is the use of a SLM to perform a real time and programmable control of the fringe visibility of two SC sources.

The experimental setup for measuring the coherence of adjacent SC pulses is shown in Fig. 3. As a light source we used the same Ti:Sapphire femtosecond laser than in the previous experiment. Before the laser beam impinges onto a reflective liquid crystal on silicon phase-only SLM (Holoeye, Pluto), it was conveniently attenuated with neutral filters. The SLM has $1920 \times 1080$ pixels with $8 \mu \mathrm{m}$ of pixel pitch. Two diffractive microlenses (DMLs) of $1.44 \times 1.44 \mathrm{~mm}^{2}$ each, were encoded onto the SLM both with a focal length of $60 \mathrm{~mm}$ for the central wavelength of the laser. The phase of each DML corresponds to that of a kinoform profile given by [25]:

$$
\varphi\left(r, f_{j}\right)=\bmod \left(\frac{\pi r^{2}}{\lambda_{0} f_{j}}, 2 \pi\right)
$$

where j-index refers to each DML, $\lambda_{0}$ is the central wavelength, $f_{j}$ is the focal length and $r$ is the radial coordinate. The function $\bmod (x, y)$ gives the remainder on division of $x$ by $y$. This phase ranges from 0 to $2 \pi$. In order to reduce the foci's lateral separation, two opposite linear phases of the same period (80 $\mu \mathrm{m})$ were overlapped to each of the DMLs. In this way, two filaments were formed, with a lateral separation of $0.2 \mathrm{~mm}$.

Next, the reflected beam from the SLM passed through a 1x telescope composed of a pair of lenses $L_{1}$ and $L_{2}$ of focal lengths $\mathrm{f}_{1}=\mathrm{f}_{2}=100 \mathrm{~mm}$. The telescope imaged the SLM plane between the lens $\mathrm{L}_{2}$ and a $5 \mathrm{~mm}$ thick fused silica block used for SC generation. The fused silica block was placed at the focal plane of the DMLs. In this way, two separated filaments were formed. The total incident energy before the beam-splitter was risen up to $110 \mu \mathrm{J}$. Due to the losses in the beam-splitter and the SLM, we estimate a $3.5 \%$ of the total incident energy per kinoform lens reaching the sample. A side view of the filaments profile was made through a lens $(\mathrm{f}=60 \mathrm{~mm})$ and registered by a camera. The straight forward SC light was projected onto a CCD camera which had an integrated band-pass filter (Thorlabs, FGB37S) to remove the non-converted infrared beam.

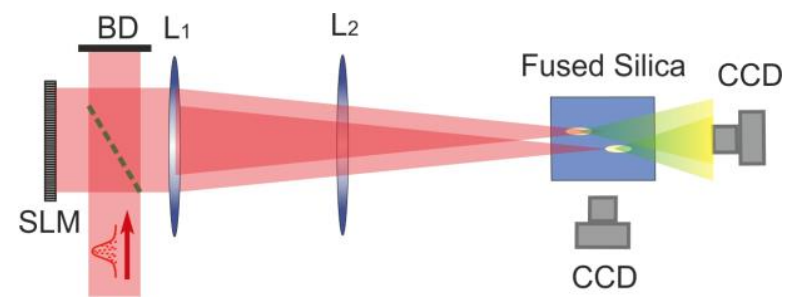

Fig. 3. Experimental setup with the SLM. (BD: beam dumper; $\mathrm{L}_{1}$ and $\mathrm{L}_{2}$ : refractive lenses).

Although the delay between the two sources cannot be controlled with this scheme, the use of the SLM presents several 
advantages with respect to the interferometer: (i) it allows for real time and programmable control of the fringes; (ii) it is easy to align; (iii) as it will be demonstrated in the next section, we can account for the effect of beam inhomogeneities; (iv) multiple SC sources can be easily obtained by codifying a matrix of lenses. In particular, the latter, would be a rather complicated setup if an interferometer was employed.

\section{RESULTS AND DISCUSSION}

Under the above conditions, two filaments were formed, as shown in Fig. 4a. In spite of using the same focal length for both DMLs, the filaments appeared at different depths of the sample (separation of $\sim 640 \mu \mathrm{m}$ ), which resulted in a minimal fringe visibility, as expected from the experiments shown in Sect. 2. This focal offset can be attributed to beam inhomogeneities in terms of amplitude and phase, which may eventually lead to different position and length of the filaments. The wavefront of our laser was measured and no significant wavefront distortions were observed. However, the non-homogeneous intensity beam profile yielded an unequal energy distribution among both DMLs. As a direct consequence, the beam carrying more energy self-focused closer to the sample's front face. So although this setup is more compact than the interferometric one, it cannot be directly used to generate highly correlated SC beams unless this problem is solved. a)
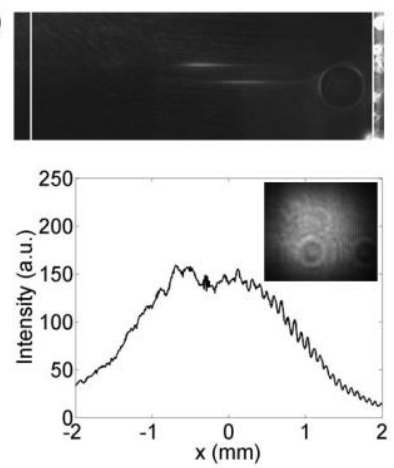
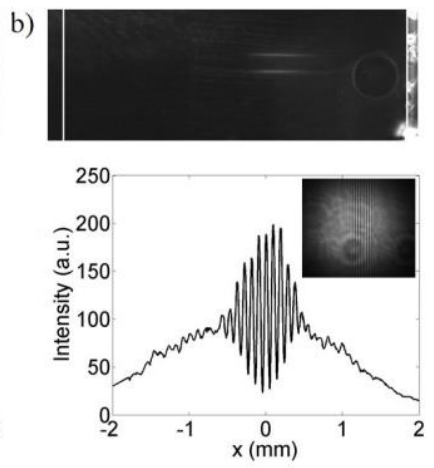

Fig. 4. Pictures of the filaments' profile (top) and corresponding fringe profile (bottom) a) without and b) with efficiency compensation Inset: interferogram. c) Visibility of the central fringe as a function of the focal length of one of the DMLs.

To face this drawback and to gain control over the energy coupled to the focus, in our proposal the phase encoded into the SLM (Eq. (2)) was multiplied by the design parameter $\alpha_{\mathrm{j}}$ :

$$
\psi\left(r, f_{j}, \alpha_{j}\right)=\alpha_{j} \varphi\left(r, f_{j}\right)
$$

with $\alpha_{j}$ from 0 to 1 . It can be demonstrated that the efficiency $\eta$ of the lens, and thus the energy coupled to the focus, is related to $\alpha$ through the expression [30]:

$$
\eta=\operatorname{sinc}^{2}(1-\alpha)
$$

By adjusting the design parameter, $\alpha$, and consequently the efficiencies of the DMLs, the energies coupled to both foci were equalized and thus the fringe contrast was increased. To do this, we first determined the energy thresholds to generate SC with each DML which were found to be different $(3.2 \mu \mathrm{J}$ for the $\mathrm{DML}_{1}$ and $2.5 \mu \mathrm{J}$ for $\mathrm{DML}_{2}$ ). Thus the efficiency of $\mathrm{DML}_{1}$ was reduced to $80 \%(\alpha=0.75)$, while it was kept to $100 \%(\alpha=1)$ for
$\mathrm{DML}_{2}$, ensuring that the energy coupled to both foci was similar. Fig. $4 \mathrm{~b}$ shows the image of the filaments with efficiency compensation and the corresponding interferogram. Now, clear fringes are observed and we obtained a fringe visibility of 0.78 in good agreement with the expected maximum value measured with the Mach-Zehnder interferometer. Therefore, just by changing the $\alpha$ parameter we can tune the maximum fringe visibility obtained with our SLM proposal.

To provide a more exhaustive control of the fringe visibility at a particular point of space, we performed a study of the fringe visibility as a function of the filaments' relative position. This was done by changing the focal length of $\mathrm{DML}_{2}$. In Fig. 5 we depicted the visibility of the fringe located at $\mathrm{x}=0$ (the center of the two beams) as a function of the focal length of $\mathrm{DML}_{2}$. As expected, once the suitable $\alpha$ parameter was encoded in both lenses, the highest coherence degree was found for $\mathrm{f}=60 \mathrm{~mm}$ (i.e., same focal length for both DMLs) achieving a visibility of 0.78 , and decreased monotonically as the filaments were progressively separated. In this case, the maximum visibility was displaced through positive values of the coordinate $\mathrm{x}$ where both pulses overlapped in time. However the poor temporal overlapping at $\mathrm{x}=0$ allowed us to tune in real time the desired visibility in this position.

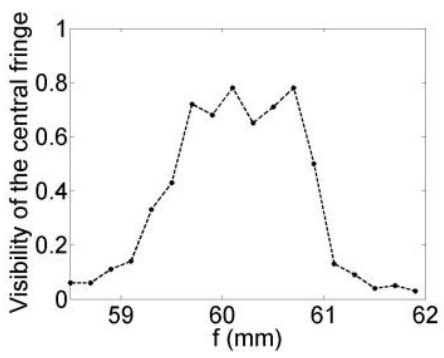

Fig. 5. Visibility of the central fringe as a function of the focal length of one of the DMLs.

\section{CONCLUSIONS}

DLs have been shown to be a powerful tool for the spectral control of SC generation in bulk [31-33]. Here, we have demonstrated the dynamic control of the fringe visibility obtained by the interference between SC pulses generated in bulk media by using DMLs encoded into a SLM. First, the careful tuning of the DML's efficiency allowed for the optimization of the maximum fringe visibility. On the other hand, by changing the focal length of each DML, we can control easily the fringe visibility at a specific point of space. Thus, the use of a SLM allows for the implementation of compact and robust setups, with large control capabilities in the fringe visibility. The results for the SLM setup are in good agreement with the measurements obtained with the interferometric setup. Moreover, the easy alignment and operation of these systems overcome the problems of the tedious interferometric setups. We believe that our proposal could be extended to filamentation in gases. In this case, its application is mainly limited by the damage threshold of the SLM. Moreover, the ability to control 
multiple filaments might be useful for energy up scaling by, for example, filament coalescence [34].

\section{REFERENCES}

[1] R.R. Alfano, The Supercontinuum Laser Source (Springer, 2006).

[2] R.R. Alfano and S. L. Saphiro, "Emission in the region 4000 to $7000 \AA$ Via Four-Photon Coupling in Glass," Phys. Rev. Lett. 24, 584-587 (1970).

[3] J. M. Dudley, G. Genty, and S. Coen, "Supercontinuum generation in photonic crystal fiber", Rev. Mod. Phys. 78, 1135-1184 (2006).

[4] V. I. Klimov and D. W. McBranch, "Femtosecond high-sensitivity, chirpfree transient absorption spectroscopy using kilohertz lasers," Opt. Lett. 23, 277-279 (1998).

[5] Z. Wilkes, S. Varma, Y.-H. Chen, H. Milchberg, T. Jones and A. Ting, "Direct measurements of the nonlinear index of refraction of water at 815 and $407 \mathrm{~nm}$ using single-shot supercontinuum spectral interferometry, "' Appl. Phys. Lett. 94, 211102 (2009).

[6] G. Cerullo and S. de Silvestri, "Ultrafast optical parametric amplifiers," Rev. Sci. Instrum. 74, 1-18 (2003).

[7] A. Couairon, A. Mysyrowicz, "Femtosecond filamentation in transparent media," Phys. Reports 441, 47-189 (2007).

[8] C.P. Hauri, W. Kornelis, F.W. Helbing, A. Heinrich, A. Couairon, A. Mysyrowicz, J. Biegert, and U. Keller, "Generation of intense, carrierenvelope phase-locked few-cycle laser pulses through filamentation," Appl. Phys B 79, 673-677 (2004).

[9] I. Hartl, X. D. Li, C. Chudoba, R. K. Ghanta, T. H. Ko, J. G. Fujimoto, J. K. Ranka and R. S. Windeler, "Ultrahigh-resolution optical coherence tomography using continuum generation in an air-silica microstructure optical fiber," Opt. Lett. 29, 608-610 (2001).

[10] S. A. Diddams, D. J. Jones, J. Ye, S. T. Cundiff, and J. L. Hall, "Direct Link between Microwave and Optical Frequencies with a $300 \mathrm{THz}$ Femtosecond Laser Comb," Phys. Rev. Lett. 84, 5102-5105 (2000).

[11] H. Takara, "Multiple optical carrier generation from a supercontinuum source," Opt. Photonics News 13, 48-51, (2002).

[12] A. Baltuška, T. Fuji, and T. Kobayashi, "Visible pulse compression to 4 fs by optical parametric amplification and programmable dispersion control," Opt. Lett. 27, 306-308 (2002).

[13] T. Udem, J. Reichert, R. Holzwarth, and T. W. Hänsch, "Absolute optical frequency measurement of the Cesium D1 line with a mode-locked laser," Phys. Rev. Lett. 82, 3568-3571 (1999).

[14] X. P. Jiang, M. Shapiro, and P. Brumer, "Pump-dump coherent control with partially coherent laser pulses," J. Chem. Phys. 104, 607 (1996).

[15] J.M. Dudley and S. Coen, "Coherence properties of supercontinuum spectra generated in photonic crystal and tapered optical fibers" Opt. Lett. 27, 1180-1182 (2002).

[16] G. Genty, M. Surakka, J. Turunen, and A.T. Friberg, "Second-order coherence of supercontinuum light”, Opt. Lett. 35, 3057-3509 (2010).

[17] G. Genty, M. Surakka, J. Turunen, and A.T. Friberg, "Complete characterization of supercontinuum coherence" J. Opt. Soc. Am. B 28, 2301-2309 (2011)

[18] M. Bellini and T. W. Hänsch, "Phase-locked white-light continuum pulses: toward a universal optical frequency-comb synthesizer," Opt. Lett. 25, 1049-1051 (2000).

[19] I. Zeylikovich and R. R. Alfano, "Coherence properties of the supercontinuum source," Appl. Phys. B 77, 265-268 (2003).

[20] C. Corsi, A. Tortora and M. Bellini, "Mutual coherence of supercontinuum pulses collinearly generated in bulk media," Appl. Phys. $B$ 77, 285-290 (2003).

[21] W. Watanabe, Y. Masuda, H. Arimoto and K. Itoh, "Coherent array of white-light continuum generated by microlens array," Opt. Review 6, 167172 (1999)

[22] P. Baum, S. Lochbrunner, J. Piel and E. Riedle, "Phase-coherent generation of tunable visible femtosecond pulses," Opt. Lett. 28, 185-187 (2003).

[23] M. Bradler, P. Baum and E. Riedle, "Femtosecond continuum generation in bulk laser host materials with sub- $\mu \mathrm{J}$ pump pulses," Appl. Phys. B 97, 561-574 (2009).

[24] C. Corsi, A. Tortora and M. Bellini, "Generation of a variable linear array of phase-coherent supercontinuum sources," Appl. Phys. B 78, 299-304 (2004).
[25] K. Cook, R. McGeorge, A. K. Kar, M. R. Taghizadeh and R. A. Lamb, "Coherent array of white-light continuum filaments produced by diffractive microlenses," Appl. Phys. Lett. 86, 021105 (2005).

[26] N, Šiauly, A. Melninkaitis, and A. Dubietis, "In situ study of two interacting femtosecond filaments in sapphire," Opt. Lett. 40, 2285-2288 (2015).

[27] K. Stelmaszczyk, P. Rohwetter, Y. Petit, M. Fechner, J. Kasparian, J. P. Wolf, and L. Woeste, "White-light symmetrization by the interaction of multifilamenting beams," Phys. Rev. A 79, 053856 (2009).

[28] J. Kasparian, M. Rodriguez, G. Méjean, J. Yu, E. Salmon, H. Wille, R. Bourayou, S. Frey, Y. B. André, A. Mysyrowicz, R. Sauerbrey, J. P. Wolf, and L. Wöste, "White-light filaments for atmospheric analysis," Science, 301, 61-64 (2003).

[29] Born \& Wolf, Principles of Optics (Cambridge University Press, Cambridge, 2006).

[30] V. Moreno, J. F. Roman, and J. R. Salgueiro, "High efficiency diffractive lenses: Deduction of kinoform profile," American Journal of Physics 65, 556-562 (1997)

[31] C. Romero, R. Borrego-Varillas, A. Camino, G. Mínguez-Vega, O. Mendoza-Yero, J. Hernández-Toro, and J. R. Vázquez de Aldana, "Diffractive optics for spectral control of the supercontinuum generated in sapphire with femtosecond pulses," Opt. Express 19, 4977-4984 (2011).

[32] R. Borrego-Varillas, C. Romero, O. Mendoza-Yero, G. Mínguez-Vega, I. Gallardo and J. R. Vázquez de Aldana, "Femtosecond filamentation in sapphire with diffractive lenses," J. Opt. Soc. Am. B 30, 2059-2065 (2013).

[33] R. Borrego-Varillas, J. Pérez-Vizcaíno, O. Mendoza-Yero, G. MínguezVega, J. R. Vázquez de Aldana and J. Lancis, "Controlled multi-beam supercontinuum generation with a spatial light modulator," Phot. Tech. Lett., IEEE, 26, 1661-1664 (2014).

[34] S. Tzortzakis, L. Bergé, A. Couairon, M. Franco, B. Prade, and A. Mysyrowicz, "Breakup and Fusion of Self-Guided Femtosecond Light Pulses in Air," Phys. Rev. Lett. 86, 5470 (2011). 